\title{
Monolayer Studies on Adsorbed Layer Thickness of Polyvinylpyrrolidone on Polystyrene Latices and Steric Stabilization Free Energy
}

\author{
Masami KAWAGUCHI, Itsuki HAYASHI, and Akira TAKAHASHI \\ Department of Industrial Chemistry, Faculty of Engineering, \\ Mie University, Kamihama-cho, Tus, Mie 514, Japan.
}

(Received February 10, 1981)

\begin{abstract}
Polystyrene latex particles and polystyrene latices covered by adsorbed polyvinylpyrrolidones were spread out as a particle thick layer over the interface between air and an aqueous $2 \mathrm{~N} \mathrm{NaCl}$ solution, and the surface pressures of the latices were measured by a surface balance. The thickness of polyvinylpyrrolidone adsorbed on the latices was evaluated from the surface area where the surface pressures were first detected. The thickness of polyvinylpyrrolidone was several times larger than the root-mean-square end-to-end distance of the isolated free coil in solution, showing considerable extension of adsorbed polymers normal to the surface of the polystyrene latex. The repulsive free energy of polystyrene latices covered by adsorbed polyvinylpyrrolidone layer was evaluated from the surface pressure-area isotherms. This was observed even at the theta point, indicating that the volume restriction free energy term is operative, since the aqueous $2 \mathrm{~N} \mathrm{NaCl}$ solution was found to be a theta solvent for polyvinylpyrrolidone. The observed repulsive free energy was compared with the theory of Hesselink, Vrij, and Overbeek. The measured repulsive free energy was in agreement with the volume restriction free energy calculated by a model of equal tails. It was concluded that the observed large thickness is due to the existence of long tails.

KEY WORDS Surface Pressure / Polystyrene Latex / Polyvinylpyrrolidone /

Surface Balance / Thickness of Adsorbed Layer / Repulsive Free Energy /

Volume Restriction / Steric Stabilization Free Energy / Tails /
\end{abstract}

The steric stabilization of colloidal particles by interaction between adsorbed polymer layers is widely used in various industrial applications such as paints, inks, dispersions of inorganic particles and emulsions as well as polymer latices. Indeed this technique was employed by the Egyptians to prepare inks as early as 4,500 years ago.

Theories of steric stabilization originated by the adsorbed polymer layers have been presented by Mackor, ${ }^{1}$ Fisher, ${ }^{2}$ Clayfield and Lumb, ${ }^{3}$ Meier, ${ }^{4}$ Hesselink, Vrij, and Overbeek, ${ }^{5,6}$ Evans and Napper, ${ }^{7}$ and Dolan and Edwards. ${ }^{8}$ According to Evans and Napper, ${ }^{7}$ the interparticle interaction free energy makes two kinds of contributions, i.e., the osmotic term and the van der Waals attraction term, while, according to Meier, ${ }^{4}$ and Hesselink, Vrij, and Overbeek (HVO), ${ }^{6}$ there are three contributions, namely the osmotic, the volume restriction, and the van der Waals interactions. Since the osmotic repulsive term disappears at the theta point, Evans and Napper ${ }^{7}$ claim that the theta condition corresponds to the limit of stability of stericaly stabilized colloidal dispersions. Moreover, Napper and his coworkers ${ }^{9-12}$ showed from their experiments that a stable dispersion could not be obtained under theta conditions.

The volume restriction repulsion between adsorbed polymer layers originates from the loss of conformational freedom of the adsorbed chains and thus does not disappear even at the theta condition. Using the surface balance technique, Doroszkowski and Lambourne ${ }^{13}$ measured the surface pressure of polyacrylonitrile dispersions stabilized by polystyrene chains at the interface of cyclohexane-water as well as toluene-water, and observed a repulsive force even stronger than under the theta condition, and concluded that the volume restriction term was operative. Moreover, they suggested that the larger thickness of adsorbed polystyrene layer was dominated by long tails.

Lyklema and Vliet ${ }^{14,15}$ measured both the thickness of free liquid films stabilized by adsorbed 
poly(vinyl alcohol) or partially esterified poly(methacrylic acid) and the disjoining pressure, and showed that the thickness of adsorbed polymer layers exceeded the values determined by ellipsometry and arrived at the conclusion that long tails dominate this large observed thickness of the adsorbed layer.

Recently, Gavey, Mitchell, and Smith ${ }^{16}$ succeeded in spreading polystyrene latices stabilized by adsorbed polyvinylpyrrolidone over the interface between air and an aqueous $2 \mathrm{~N} \mathrm{NaCl}$ solution, measured the surface pressure, and showed that the thickness of polyvinylpyrrolidone adsorbed onto latices is much larger than the diameter of a free chain in solution. However, they did not measure the steric stabilization free energy from pressure-area isotherms.

According to recent polymer adsorption theories, steric stabilization free energy is expressed as a function of both the interparticle distance and the loop or tail size, depending on the assumed conformation of adsorbed polymer chains, i.e., the looptrain model or the loop-train-tail model. Therefore, to compare these theories with experiment, it is necessary not only to determine the adsorbed layer thickness but also to measure the interparticle interaction free energy. In this respect, the surface balance technique pioneered by Doroszkowski and Lambourne, ${ }^{13,17}$ is very suitable.

The purpose of this paper is first to determine the conformation of polyvinylpyrrolidone adsorbed onto polystyrene latices and second, to measure the interparticle free energy and compare it with the existing theories to clarify whether the volume restriction term is operative even under the theta condition.

\section{EXPERIMENTAL}

\section{Materials}

Surfactant free polystyrene latices were prepared by an emulsion polymerization technique under nitrogen atmosphere at $70^{\circ} \mathrm{C}$ using potassium persulphate as an initiator. After polymerization, the latices were filtered through a glass filter $3 \mathrm{G}-1$ to remove any coagulums formed and then, unreacted monomers were removed by steam distillation. The latices were dialyzed against distilled water for several weeks and further purified by an Amberlite mixed resin. The polystyrene content in the latices was measured by weighing after a complete evaporation of water. The average particle size of the latex was determined by measuring the diameter of latex particles on an electron micrograph and found to be $5600 \pm 100 \AA$.

Commercial polyvinylpyrrolidone (hereafter abbreviated PVP) obtained from Nakarai Chemical Co. was fractionated at $25^{\circ} \mathrm{C}$ from its aqueous solution using acetone as a precipitant. Each fraction was dissolved in methanol, precipitated by ethyl acetate, and then freeze-dried from an aqueous solution. The intrinsic viscosities of the fractionated PVP samples in an acetone-water mixture (vol ratio 66.8/33.2) and in aqueous $2 \mathrm{~N} \mathrm{NaCl}$ solution at $25^{\circ} \mathrm{C}$ were measured by an Ubbelohde viscometer. The molecular weights of the fractionated PVP samples were calculated from the intrinsic viscosities in the acetone-water mixture according to the following equation, ${ }^{18}$

$$
[\eta]=75 \times 10^{-5} M^{0.5}
$$

Characteristics of PVP samples are summarized in Table I.

Table I. Characteristics of polyvinylpyrrolidones

\begin{tabular}{ccccc}
\hline & & {$[\eta]_{\mathrm{aq} .2 N \mathrm{NaCl}}$} & & $\left\langle r^{2}\right\rangle^{1 / 2} \times 10^{-2}$ \\
\cline { 5 - 5 } Samples & $M \times 10^{-4}$. & $\mathrm{dl} \mathrm{g}^{-1}$ & & $\AA$ \\
\hline PVP-1 & $8.7_{6}$ & $0.22_{1}$ & & $1.8_{9}$ \\
PVP-2 & 19.6 & $0.35_{0}$ & & $2.8_{8}$ \\
PVP-3 & $72 .{ }_{6}$ & $0.61_{6}$ & & $5.3_{8}$ \\
\hline
\end{tabular}

Extra pure grade sodium chloride was used without further purification.

Water was doublely distilled by all pyrex apparatus.

\section{Adsorption of PVP onto Polystyrene Latices}

Seven mililiters of aqueous PVP solution with known concentration were added to five mililiters of the original polystyrene latices in a stoppered test tube and gentely stirred by a magnetic chip at $25^{\circ} \mathrm{C}$. No flocculation of latices was observed for any sample. After $48 \mathrm{~h}$, the latices were centrifuged at $2 \times 10^{4} \mathrm{~g}$ for $30 \mathrm{~min}$ to sediment the latices and then the supernatant was carefully removed. To remove the unadsorbed PVP, the sedimented latices were redispersed in distilled water, recentrifuged, and the supernatant was separated. This procedure was 
repeated five times. All separated supernatants were collected and the concentration of PVP in the collected supernatants was measured by a UV spectrometer (Union Giken system 77) at the wavelength $200 \mathrm{~nm}$. The relevent concentration of PVP was determined from a calibration curve. The adsorbed amounts, $A$ were determined from the difference between the amounts of initially added PVP and those in the collected supernatants.

\section{Surface Pressure Measurements}

Naked polystyrene latices as well as latices covered by PVP were spread over the air-aqueous $2 \mathrm{~N}$ $\mathrm{NaCl}$ solution interface. A Langmuir-Adam type surface balance equipped with a mica float coated with paraffin was used for compression studies of latices. Measurements were carried out at $25^{\circ} \mathrm{C}$. Both naked and PVP covered latices were redispersed in distilled water by ultrasonic radiation before spreading over the air- $2 \mathrm{~N} \mathrm{NaCl}$ solution interface. A tefion trough was filled with an aqueous $2 \mathrm{~N} \mathrm{NaCl}$ solution on which latices could be spread as a monolayer since the density of aqueous $2 \mathrm{~N} \mathrm{NaCl}$ solution is greater than that of polystyrene. For the naked latices, the addition of 2-propanol was required to assist the spreading, while for the latices covered by PVP, this was unnecessary. The surface pressure was continuously measured from successive changes of area by moving a teflon barrier, and the accuracy of the measurement was $\pm 0.05 \mathrm{dyn}^{-1}$.

\section{RESULTS}

\section{Adsorption Isotherm of PVP onto Polystyrene Latices}

The adsorption isotherm of PVP-1 with molecular weight of $8.76 \times 10^{4}$ is shown in Figure 1. The adsorbed amount, $\mathrm{A}$, increases with increasing bulk
PVP concentration and reaches a plateau region at a PVP concentration of about $0.3 \mathrm{~g} 100 \mathrm{ml}^{-1}$. The same trends were also observed for other PVP samples. The adsorbed amounts at a bulk PVP concentration of about $0.5 \mathrm{~g} / 100 \mathrm{ml}$, well within the plateau region, are listed in Table II and decrease with increasing molecular weight.

\section{Surface Pressure-Area Isotherms of Latices}

The surface pressure-area isotherms of the naked polystyrene latices and the latices covered by PVP-1 adsorbed at a bulk PVP concentration of $0.51 \mathrm{~g} / 100 \mathrm{ml}$ are shown in Figure 2. The surface pressure of the naked polystyrene latices gradually increases with decreasing surface area and then sharply increases. Above the surface pressure of $5 \mathrm{dyn} \mathrm{cm}^{-1}$ the polystyrene latex monolayer shows irridescent color, indicating that the polystyrene latex particles were not only monodisperse in size as evidenced by the electron micrograph but also closely packed at the interface (Figure 3 ). To examine the state of packing of the polystyrene latices at the air-aqueous $2 \mathrm{~N} \mathrm{NaCl}$ solution interface, the latex film at the surface pressure of $5 \mathrm{dyn}^{-1}$ was transferred onto a mesh of electron microscopy.

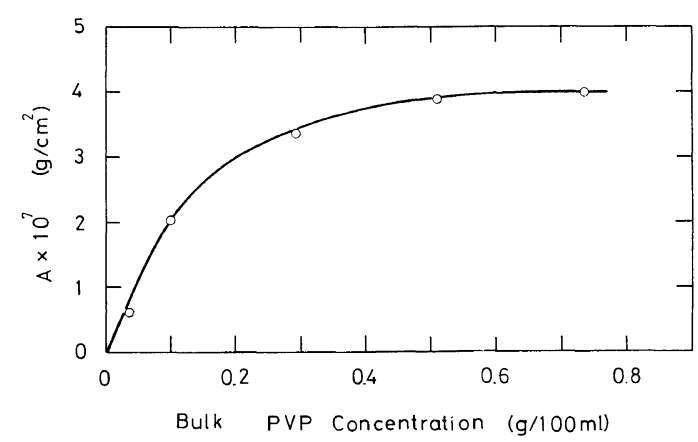

Figure 1. Adsorption isotherm of PVP-1 adsorbed on the polystyrene latices at $25^{\circ} \mathrm{C}$.

Table II. Bulk PVP concentration, $C_{\mathrm{p}}$, adsorbed amount, $A$, thickness of adsorbed PVP layer, $\delta$, and observed repulsive free energy per contact point between latex particles, $\Delta F_{\text {obs. }}$, of latices covered by PVP samples

\begin{tabular}{|c|c|c|c|c|}
\hline \multirow{2}{*}{ Latices } & $C_{\mathrm{p}}$ & $A \times 10^{7}$ & $\delta \times 10^{-3}$ & $\Delta F_{\text {obs. }} \times 10^{10}$ \\
\hline & $\mathrm{g} / 100 \mathrm{ml}$ & $\mathrm{g} \mathrm{cm}^{-2}$ & $\AA$ & erg \\
\hline SL-PVP-1 & 0.51 & 3.8 & 1.35 & 3.8 \\
\hline SL-PVP-2 & 0.51 & 3.7 & 1.66 & 2.8 \\
\hline SL-PVP-3 & 0.52 & 1.7 & 1.98 & 2.7 \\
\hline
\end{tabular}




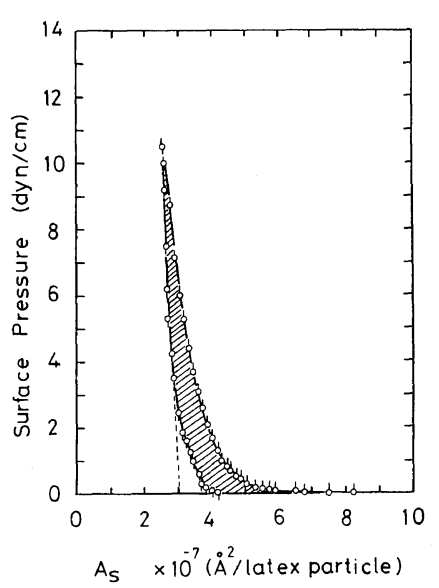

Figure 2. Surface pressure-area isotherm of the naked polystyrene latex particles $(O)$ and the polystyrene latices covered by PVP-1 ( $\bigcirc$ ) at the interface between air and the aqueous $2 \mathrm{~N} \mathrm{NaCl}$ solution. The dotted line indicates the linear extraporation of the surface pressure-area isotherm for the naked polystyrene latices to zero surface pressure.

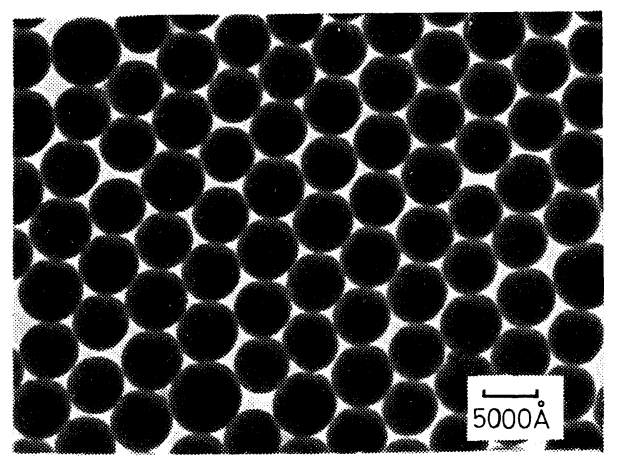

Figure 3. Electron micrograph of close packed latex monolayer.

The electron micrograph of Figure 3 shows that the polystyrene latex particles are nearly hexagonally close-packed in two dimensional array, i.e., a particle thick monolayer, though, somewhat incomplete packing can be observed. A limiting surface area was obtained as $3.01 \times 10^{7} \AA^{2} /$ latex particle by linear extraporation of the surface pressure-area isotherm to zero surface pressure. From this value the diameter of polystyrene latex particle was calculated to be $5900 \AA$, assuming that the latices are hexagonaly closed-packed. This value is a little larger than the diameter of $5600 \AA$ determined by electron micrography. The difference can be attributed to the incomplete packing.
The surface pressure-area isotherm for the latices covered by PVP-1 is found at a surface area larger than that of the naked polystyrene latices as shown in Figure 2. No hysteresis was observed in successive compression-expansion cycles. The area at which the surface pressure is first detected for the latices covered by PVP, can be considered an indication of the first contact of PVP chains adsorbed onto the polystyrene latices. From this area, the thickness $(\delta)$ of adsorbed PVP layer on the polystyrene latices was calculated by the following equation assuming the hexagonal closed packing of the latex particles,

$$
N \pi(r+\delta)^{2}=C A_{\mathrm{s}}
$$

where $N$ is the number of polystyrene latices injected, $r$ is the radius of a polystyrene latex particle, and taken to be $2800 \AA, C$ is the two dimensional packing factor, taken to be 0.907 , and $A_{\mathrm{s}}$ is the surface area at which the surface pressure becomes detectable. The values of $\delta$ calculated from eq 2 are summarized in Table II and increase with increasing molecular weight.

The total repulsive free energy can be calculated from the graphical integration of the area of the hatched region shown in Figure 2. The repulsive free energy per contact point between particles was calculated by dividing the total repulsive free energy by $6 N$, since in the hexagonal close packing there are six contact points around each particle. The resulting value are listed in Table II. The repulsive free energy per latex particle decreases with increasing molecular weight.

The intrinsic viscosities of the PVP samples in aqueous $2 \mathrm{~N} \mathrm{NaCl}$ solution at $25^{\circ} \mathrm{C}$ are plotted against molecular weight on logarithmic scale in Figure 4 . The following viscosity equation can be

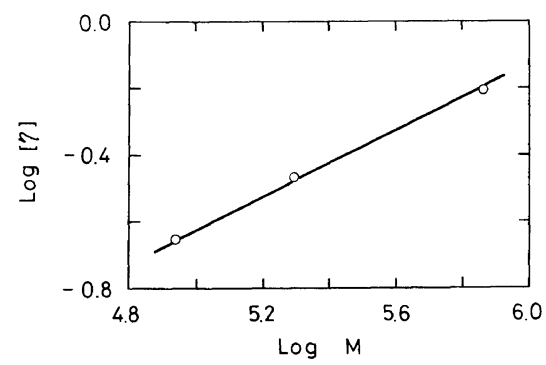

Figure 4. Plots of intrinsic viscosities of PVP in aqueous $2 \mathrm{~N} \mathrm{NaCl}$ solution against the molecular weights. 
derived:

$$
[\eta]_{\text {aq. } 2 \mathrm{~N} \mathrm{NaCl}}=7.45 \times 10^{-4} M^{0.5}
$$

This equation indicates that aqueous $2 \mathrm{~N} \mathrm{NaCl}$ is a theta solvent for PVP.

\section{DISCUSSION}

\section{Layer Thickness of Adsorbed PVP}

The layer thickness of adsorbed PVP and the root-mean-square end-to-end distance, $\left\langle r^{2}\right\rangle^{1 / 2}$, of a free polymer coil in $2 \mathrm{~N} \mathrm{NaCl}$ solution, which was calculated by the Flory-Fox equation using the intrinsic viscosity, are compared in Tables I and II. The value of $\delta$ is always larger than the root-meansquare end-to-end distance by a factor of 3 . This indicates that the adsorbed polymers are more elongated normal to the surface of polystyrene latex particle. However, the $\delta$ value is much less than the contour length of PVP. The very large values of $\delta$ for the adsorbed polymer layer are due to the existence of long tails, as explained below.

\section{Steric Stabilization Free Energy}

According to the HVO model, ${ }^{6}$ the total free energy of steric stabilization, $\Delta F_{\mathrm{T}}$, is expressed as the sum of three terms,

$$
\Delta F_{\mathrm{T}}=\Delta F_{\mathrm{M}}+\Delta F_{\mathrm{VR}}+\Delta F_{\mathrm{w}}
$$

where $\Delta F_{\mathrm{M}}$ is related to the osmotic repulsion, $\Delta F_{\mathrm{VR}}$ with the volume restriction repulsion, and $\Delta F_{\mathrm{w}}$ with the van der Waals attraction. The first term, originating from the overlap of the adsorbed polymer layer, is written as follows,

$$
\Delta F_{M}=2\left(\frac{2 \pi}{9}\right)^{3 / 2}\left(\alpha^{2}-1\right) k T v^{2}\left\langle r^{2}\right\rangle_{0} M(\bar{i}, d)
$$

where $\alpha$ is the expansion factor of an isolated polymer chain in solution, $k$ is the Boltzmann constant, $T$ is the absolute temperature, $v$ is the number of loops or tails per unit area, $\left\langle r^{2}\right\rangle_{0}$ is the mean-square end-to-end distance of the polymer chain in the unperturbed state, and $M(\bar{i}, d)$ is given as follows. For equal loops

$$
M(\bar{i}, d)=(3 \pi)^{1 / 2}\left[6 d^{2} /\left(\bar{i} l^{2}\right)-1\right] \exp \left(-3 d^{2} / \bar{i} l^{2}\right)
$$

and for equal tails,

$$
M(\bar{i}, d)=(6 \pi / 5)^{1 / 2}\left[\frac{12}{5} d^{2} /\left(\left.\bar{i}\right|^{2}\right)-1\right]
$$

$$
\times \exp \left(-\frac{6}{5} d^{2} / \bar{i} l^{2}\right)
$$

where $\bar{i}$ is the average number of segments in a loop or a tail, $l$ is the segment length, and $d$ is the distance between particle surfaces. The values of $M(\bar{i}, d)$ are tabulated in ref 6 as a function of $d / \sqrt{\bar{i}} l$. The volume restriction free energy is given by

$$
\Delta F_{\mathrm{VR}}=2 v k T V(\vec{i}, d)
$$

where $V(\bar{i}, d)$ is given for equal loops,

$$
V(\bar{i}, d)=2 \exp \left(-\frac{3}{2} d^{2} / \bar{i} l^{2}\right)
$$

and for equal tails,

$$
V(\bar{i}, d)=2\left[12 d^{2} /\left(\overline{i l} l^{2}\right)-1\right] \exp \left(-6 d^{2} / \overline{i l}^{2}\right)
$$

The functions $V(\bar{i}, d)$ are also tabulated in ref 6 as a function of $d / \sqrt{\bar{i}} l$.

The van der Walls attraction free energy, $\Delta F_{\mathrm{w}}$, is expressed for two equal spherical particles as follows,

$$
\Delta F_{\mathrm{w}}=-\frac{A_{H}}{6}\left[\frac{2 r^{2}}{H^{2}-4 r^{2}}+\frac{2 r^{2}}{H^{2}}+\ln \left(1-\frac{4 r^{2}}{H^{2}}\right)\right]
$$

where $A_{\mathrm{H}}$ is the Hamaker constant, $H$ is the distance between the centers of spherical particles with a radius of $r$. Since $A_{\mathrm{H}}=3 \times 10^{-14} \mathrm{erg}$ for polystyrene, ${ }^{19,20} \Delta F_{\mathrm{w}}$ for $r=2800 \AA$ is calculated to be $-1.47 \times 10^{-14}$ erg at $H=6000 \AA$, namely $d / 2=200 \AA$. This van der Waals attraction free energy is much smaller than the observed total repulsive free energy listed in Table II, so that $\Delta F_{\mathrm{w}}$ can be neglected. Therefore, at the theta point $\Delta F_{\mathrm{T}} \simeq F_{\mathrm{VR}}$, though, according to Evans and Napper, ${ }^{7} \Delta F_{\mathrm{T}} \simeq \Delta F_{\mathrm{M}}=0$.

Since the $2 N \mathrm{NaCl}$ solution is a theta solvent for PVP, our observation of a repulsive free energy clearly indicates that the view of Evans and Napper may be inadequate. The theory of $\mathrm{HVO}^{6}$ predicts that the dispersion is stabilized even at the theta point by the volume restriction term. The term of $\Delta F_{\mathrm{VR}}$ is controlled by loops or tails or both. To decide which portion of chains, loops or tails, govern the steric stabilization, we compared the data with the HVO theory. Since this theory for steric stabilization is only applicable to two parallel plates approaching each other, it must be corrected for the curvature of the latex particles. Following the method of Doroszkowski and Lambourne ${ }^{13}$ 


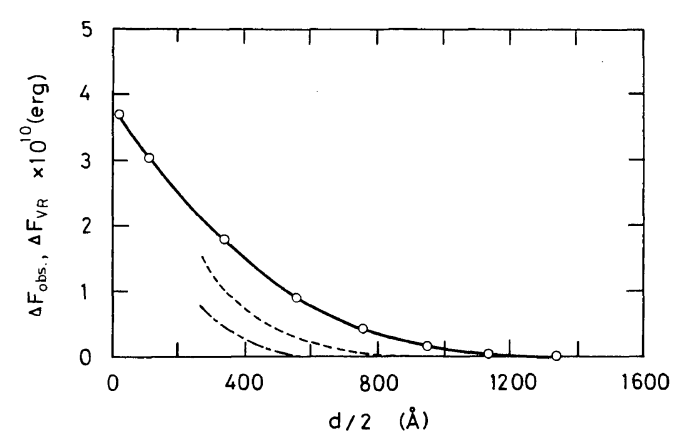

Figure 5. Comparison of the HVO theory with experimental repulsive free energy, $\Delta F_{\text {obs. }}$, for the polystyrene latices covered by PVP-1: Volume restriction free energy, $\Delta F_{\mathrm{VR}}$, calculated for equal tails (-----) and equal loops (-- -).

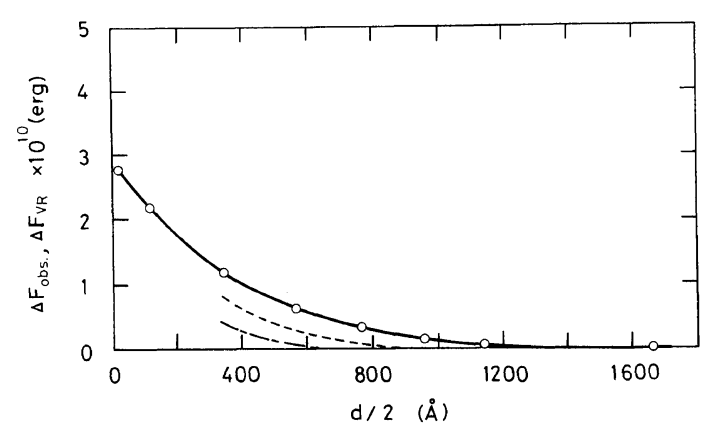

Figure 6. Comparison of the HVO theory with experimental repulsive free energy, $\Delta F_{\text {obs. }}$, for polystyrene latices covered by PVP-2. The symbols of calculated volume restriction free energy, $\Delta F_{\mathrm{VR}}$, as in Figure 5.

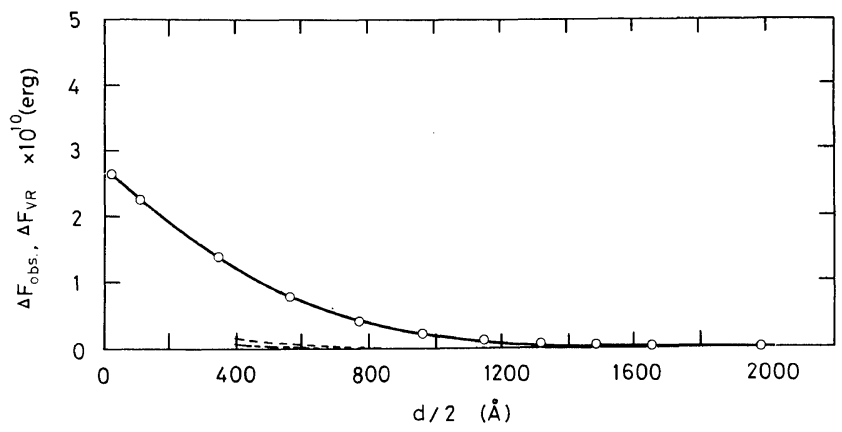

Figure 7. Comparison of the HVO theory with experimental repulsive free energy, $\Delta F_{\text {obs. }}$, for polystyrene latices covered by PVP-3. The symbols of calculated volume restriction free energy, $\Delta F_{\mathrm{VR}}$, as in Figure 5.

which was first suggested by Derjarguin, ${ }^{21}$ we treated the spherical particles as a series of concentric terraces of $15 \AA$ high. Thus, each "terrace" is considered to be a flat plate and the distance $d_{n}$ between terraces is obtained as follows,

$$
d_{n}=d+2 r\left(1-\cos \theta_{n}\right)
$$

where $\cos \theta_{n}=\sqrt{r^{2}-(15 n)^{2}} / r$ and the value of $15 n$ is equal to the radius of the planar ring parallel to each other at a distance $d_{n}$.

The value of $v$ in eq 8 is assumed to be the number of adsorbed polymers per unit area and $\sqrt{\bar{i}} l$ is put equal to $\delta$. The volume restriction free energy can be calculated using the tabulated values of $V(\bar{i}, d)$ for equal loops and equal tails. Comparisons between the experimental results and the theoretical calculations are given in Figures 5, 6 and 7 for latices covered by PVP-1, 2, and 3, respectively.

The repulsive free energies calculated on the two models of equal tails and equal loops are smaller than the observed free energies for all three samples. The calculated repulsive free energies for equal tails are not only close to the observed free energies but also appear to operate at a greater distance, $d$, than those for equal loops. Thus, the experimental repulsive free energies show qualitative agreement with those for equal tails. These results emphasize the existence of long tails, as was inferred previously from large $\delta$ values. However, the discrepancy between the calculated and measured repulsive free energies is evident from the figures. Especially, for the highest molecular weight sample, the measured repulsive free energy is one order of magnitude larger than the calculated free energy. The existence of long tails does not exclude the presence of many loops. Thus the calculated total repulsive free energy should be the sum of $\Delta F_{\mathrm{VR}(\text { tails })}$ and $\Delta F_{\mathrm{VR}(\text { loops). }}$. This sum is expected to be larger than $\Delta F_{\mathrm{VR}(\text { tails })}$ alone. Another reason for the disagreement may be the distribution of tail size, since the PVP samples 
have molecular weight distributions. At any rate, comparison between the measured and calculated compression curves confirms the view that the tail portions of adsorbed chains play a dominant role in the steric interactions of polystyrene latices covered by PVP. The latices are stabilized by the adsorbed PVP tails which extend far out. But, at a smaller distance, the contribution of loops also appears to exist. The total thickness of adsorbed PVP is governed by the long tails, while small loops and trains occur alternatively in the remaining portion of the chain, thus producing a fairly high segment density near the surface of polystyrene latices.

Acknowledgment. The authors are indebted to Mr. H. Umemoto, Plastics Development and Research Laboratories, Mitsubishi Petrochemical Company, for his assistance in taking the electron micrographs.

\section{REFERENCES}

1. E. L. Mackor, J. Colloid Sci., 6, 492 (1951).

2. E. W. Fisher, Kolloid Z., 160, 120 (1958).

3. E. J. Clayfield and E. C. Lumb, J. Colloid Interface Sci., 22, 285 (1966); Macromolecules, 1, 133 (1968).

4. D. J. Meier, J. Phys. Chem., 71, 1861 (1967).
5. F. Th. Hesselink, J. Phys. Chem., 73, 3488 (1969); 75, 65 (1971).

6. F. Th. Hesselink, A. Vrij, and J. Th. G. Overbeek, J. Phys. Chem., 75, 2094 (1971).

7. R. Evans and D. H. Napper, Kolloid Z. Z. Polym., 251, 329 and 409 (1973).

8. A. K. Dolan and S. F. Edwards, Proc. R. Soc., London, Ser. A, 343, 427 (1975).

9. D. H. Napper, Trans. Faraday Soc., 64, 1701 (1968).

10. D. H. Napper, J. Colloid Inrerface Sci., 29, 168 (1969).

11. D. H. Napper, J. Colloid Interface Sci., 32, 106 (1970).

12. D. H. Napper and A. Netschey, J. Colloid Interface Sci., 37, 528 (1971).

13. A. Doroszkowski and R. Lambourne, J. Colloid Interface Sci., 43, 97 (1973).

14. J. Lyklema and T. van Vliet, Faraday Discuss. Chem. Soc., No. 65, 25 (1978).

15. T. van Vliet, Meded Landbouwgeschoor Wageningen, The Netherlands; 77-1 (1977).

16. M. J. Gavey, D. Mitchell, and A. L. Smith, Colloid Polym. Sci., 257, 70 (1979).

17. A. Doroszkowski and R. Lambourne, J. Polym. Sci., $C$, No. 34, 253 (1971).

18. H-G. Elias, Makromol. Chem., 50, 1 (1961).

19. R. H. Ottewill and J. N. Shaw, Discuss. Faraday Soc., 42, 154 (1966).

20. M. Kawaguchi, I. Kondo, and A. Takahashi, Kobunshi Ronbunshu, 37, 427 (1980).

21. B. Derjarguin, Kolloid Z., 69, 155 (1934). 